\title{
Expresión de Calreticulina en Placenta Canina
}

\author{
Calreticulin Expression in Canine Placenta
}

Carlos Rosas C. ${ }^{1,4}$; Katherine Weinberger S. ${ }^{\text {; }}$ Ismael Maldonado F. ${ }^{2}$; David Lemus A. ${ }^{3}$ \& Arturo Ferreira V. ${ }^{2}$

\begin{abstract}
ROSAS, C. C.; WEINBERGER, S. K.; MALDONADO, F. I. ; LEMUS, A. D. \& FERREIRA, V. A. Expresión de calreticulina en placenta canina. Int. J. Morphol., 37(4):1294-1298, 2019.

RESUMEN: La placenta es un anexo embrionario de los mamíferos que tiene por función principal el intercambio de nutrientes y gases y proteger al concepto de un potencial daño inmune provocado por diferencias alogénicas en los Complejos Principales de Histocompatibilidad paternos. Se han descrito diversas proteínas asociadas a su función, siendo Calreticulina una de ellas. Si bien existen estudios de la presencia de Calreticulina en placenta humana, no existen reportes de esta proteína en la placenta canina. Se obtuvieron muestras de placenta canina de las que se extrajo el contenido proteico total y se determinó la presencia de Calreticulina por western blot e inmunohistoquímica. Los resultados mostraron presencia de Calreticulina en placenta canina con un peso molecular aparente de $60 \mathrm{kDa}$, concordante con lo descrito para la molécula por otros autores. El análisis inmunohistoquímico mostró que Calreticulina canina está presente principalmente en el trofoblasto de las vellosidades, no existiendo diferencias en cuanto a su localización al compararla con placenta humana, pese a sus diferencias morfológicas e histológicas. Esta información permitirá establecer un protocolo estandarizado de extracción de Calreticulina desde placenta, así como orientar acerca de los posibles roles de esta molécula en la placenta.
\end{abstract}

PALABRAS CLAVE: Calreticulina; Placenta; Trofoblasto.

\section{INTRODUCCIÓN}

La placenta es un anexo embrionario que se ha desarrollado en los mamíferos pertenecientes a la infraclase Eutheria y que tiene por función principal el intercambio de nutrientes y gases entre el feto y la madre y también proteger al feto de posible daño inmune mediado por diferencias alogénicas en los Complejos Principales de Histocompatibilidad materno y paterno. Está formada por una parte fetal, el corion, y una parte materna, la decidua basal, que corresponde a la zona más superficial del endometrio uterino (Roa et al., 2012; Moore et al., 2016).

Además de las funciones de intercambio, la placenta presenta una diversidad de funciones asociadas al embarazo, como la producción de hormonas polipeptídicas y esteroidales (Gudea et al., 2004) y la modulación de la respuesta inmune ante el embrión, con el fin de evitar el rechazo de este "aloinjerto" por el sistema inmune materno (Moffett \& Loke, 2006).

Muchas proteínas se han identificado y asociado a distintas funciones de la placenta, siendo una de ellas Calreticulina (CRT). CRT es una proteína chaperona de 47
kDa que posee una secuencia conservada en distintos organismos y es vital para la vida, ya que ratones knock-out para el gen de CRT mueren durante la gestación (Mesaeli et al., 1999). Se localizó inicialmente en el retículo endoplásmico, donde controla los niveles de calcio y regula el correcto plegamiento de proteínas (Gelebart et al., 2005). Más tarde, se descubrió que CRT es también importante para el correcto ensamblaje de Moléculas de Histocompatibilidad de clase I, la adhesión y migración celular, la comunicación célulacélula, la fagocitosis y la apoptosis, destacando su rol en otros procesos fisiológicos y patológicos como la cicatrización de heridas y el cáncer (Gelebart et al.; Gold et al., 2010). CRT ha sido aislada desde músculo (Flieguel et al., 1989), hígado (Milner et al., 1991), placenta humana (Houen \& Koch, 1994), torrente sanguíneo (Michalak et al., 1999) y corazón (Mesaeli et al.), además de expresarse en la superficie de distintos mamíferos, insectos y parásitos (Arosa et al., 1999; Michalak et al.).

Recientemente, CRT se ha aislado desde endometrio murino y se ha descrito que los niveles máximos de esta proteína coinciden con el momento de la implantación del

\footnotetext{
${ }^{1}$ Departamento de Ciencias Morfológicas, Facultad de Medicina y Ciencia, Universidad San Sebastián, Santiago, Chile.

${ }^{2}$ Programa Disciplinario de Inmunología, Instituto de Ciencias Biomédicas, Facultad de Medicina, Universidad de Chile, Santiago, Chile.

${ }^{3}$ Programa de Anatomía y Biología del Desarrollo, Instituto de Ciencias Biomédicas, Facultad de Medicina, Universidad de Chile, Santiago, Chile.

${ }^{4}$ Núcleo Ciencias Biológicas, Facultad de Estudios Interdisciplinarios, Universidad Mayor, Santiago, Chile.
} 
blastocisto (Cheng et al., 2009). Estudios en paralelo han reportado niveles elevados de CRT en sangre de mujeres embarazadas versus no embarazadas (Gu et al., 2008), y niveles aún más elevados en mujeres con ciertas patologías del embarazo como pre-eclampsia (Crawford et al., 2012). De igual manera, se ha visto que mujeres con preeclampsia poseen bajos niveles de calcio (Kisters et al., 2000), lo que sería consistente con el hecho de que CRT al encontrarse elevada en estos casos mantendría calcemias disminuidas. Por otra parte, CRT humana media el contacto inicial de Trypanosoma cruzi (agente de la enfermedad de Chagas) con el sinciciotrofoblasto, en forma dependiente del primer componente $\mathrm{C} 1$ del Complemento, preámbulo necesario para infección congénita del feto (Castillo et al., 2013).

Si bien se ha estudiado la presencia de CRT en placenta humana, ésta no ha sido investigada en caninos (Canis lupus familiaris), considerando que pueden existir variaciones en su expresión dada las diferencias entre ambas especies. Morfológicamente, la placenta humana se clasifica como discoidal mientras que la placenta canina es de tipo zonal; histológicamente, la placenta humana es hemocorial, siendo la canina endoteliocorial. Dado que en placenta humana se han detectado altos niveles de CRT (Højrup et al., 2001), la placenta canina podría ser una fuente importante de esta proteína para estudios futuros en el área, considerando que es un tejido más accesible.

Aquí describimos la presencia de CRT en placenta canina, proponemos un protocolo estandarizado para la obtención de esta proteína y determinamos el patrón de expresión en la placenta. Se posibilita así el dilucidar el rol de esta proteína en la preñez y en patologías en esta especiee mamíferas.

\section{MATERIAL Y MÉTODO}

Muestras de Placenta: Las placentas caninas fueron obtenidas durante cirugías rutinarias por preñeces distócicas. Se solicitó el consentimiento informado de los dueños de los animales, aunque estos especímenes son normalmente descartados. Los procedimientos realizados en los animales fueron aprobados por el Comité de Bioética sobre Investigación en Animales, Fac. de Medicina, U. de Chile (CBA\# 0560 FMUCH).

Las muestras de placenta fueron transportadas a 4 ${ }^{\circ} \mathrm{C}$ y lavadas, ambos procedimientos con suero fisiológico, se removió el tejido fibroso, se extrajeron las proteínas y se las procesó histológicamente.
Extracción Proteica. Las proteínas se extrajeron desde 3 trozos de 25 gramos de 3 placentas caninas, agregando $500 \mu \mathrm{L}$ de tampón de lisis RIPA (50mM Tris-HCl, pH 7.5, 150mM $\mathrm{NaCl}, 1 \%$ Tritón X-100, $0.5 \%$ Deoxicolato sódico, $0.1 \%$ SDS), en presencia de un coctel de inhibidores de proteasas $\left(\right.$ Calbiochem $\left.{ }^{\circledR}\right)$. Se resuspendió vigorosamente y se incubó en hielo por 10 minutos. Luego, el tejido fue sonicado (MicrosonTM XL2000, Misonix), incubado 10 minutos en hielo, centrifugado a $16300 \mathrm{~g}$ por 15 minutos a $4{ }^{\circ} \mathrm{C}$ y el sobrenadante fue transferido a otro tubo. Para cuantificar la concentración de proteínas se utilizó el método de Bradford (1976).

Western blotting. Se realizó un SDS-PAGE (electroforesis en geles de poliacrilamida al $12 \% \mathrm{p} / \mathrm{v}$, en presencia de dodecil sulfato de sodio) según protocolo estándar. Se cargó $30 \mu \mathrm{g}$ de proteína total de placenta canina, además de un marcador de Peso Molecular "Page Ruler Prestained Protein Ladder" (Thermo Fisher Scientific, USA). El gel fue transferido a una membrana de nitrocelulosa, la que fue bloqueada con PBSleche al $5 \% \mathrm{p} / \mathrm{v}$ toda la noche, a $4{ }^{\circ} \mathrm{C}$. Ésta se lavó 4 veces por 5 minutos con PBS con Tween-20 (PBS-Tween) al 0,05\% v/ $\mathrm{v}$ bajo agitación y se incubó con un suero inmune policlonal anti-CRT humana en PBS-leche al $3 \%$ en dilución 1:1000, por 90 minutos a temperatura ambiente. Luego se incubó con un anticuerpo de cabra anti-conejo conjugado a fosfatasa alcalina en dilución 1:1000 en PBS-leche al $3 \%$ por 90 minutos, previo lavado de 4 veces por 5 minutos con PBS-Tween. Finalmente, las proteínas fueron reveladas usando una solución de NBT-BCIP (5-bromo-4-cloro-3-indolil fosfato/ nitroazul de tetrazolio). Las bandas obtenidas fueron escaneadas y las imágenes fueron analizadas con el Software Image J (NIH, USA), para cuantificar la densidad de banda por carril al compararlas con una curva de distintas concentraciones de CRT humana $(5,10,25$ y $50 \mathrm{ng})$. Los valores obtenidos en triplicado en ensayos independientes fueron normalizados en relación a la expresión de Beta actina y se graficaron usando el Software Graph Pad Prism $5^{\mathrm{TM}}$.

Procesamiento Histológico e Inmunohistoquímica. Para el estudio histológico se utilizó un protocolo convencional en donde se fijaron las muestras de placenta canina en formalina tamponada al $10 \%$ por 5 días, seguido de deshidratación y diafanización. Se realizaron cortes histológicos de $5 \mu \mathrm{m}$ de espesor, los que fueron teñidos con Hematoxilina-Eosina, y para la inmunohistoquímica éstos fueron hidratados, recuperados antigénicamente usando Dako Target Retrieval (CA, USA) y bloqueados en una solución de Peróxido de Hidrogeno en Metanol (1:10). Para la detección de CRT se utilizó un suero policlonal anti-CRT humana en dilución 1:1000, que fue posteriormente revelado con Histostain Plus IHC Kit, AEC, Broad Spectrum (Invitrogen, USA). Finalmente, se obtuvieron microfotografías usando una cámara Moticam 2500 (Motic, USA). 


\section{RESULTADOS}

El western blot mostró que el suero policlonal antiCRT humana reconoció a la CRT canina presente en placenta con un peso molecular aparente de $62 \mathrm{kDa}$ (Fig. 1). Dado que se cargaron $30 \mu \mathrm{g}$ de proteína total de placenta canina, se realizó un análisis densitométrico de las bandas, las que se interpolaron a una curva de CRT humana en distintas concentraciones. El análisis arrojó que CRT representa aproximadamente el $0,1 \%$ de las proteínas placentarias totales extraíbles con el procedimiento descrito.
En el análisis histológico, la placenta canina mostró la arquitectura normal de una placenta de tipo endoteliocorial, observándose los vasos sanguíneos maternos en íntimo contacto con la vellosidad fetal revestida por trofoblasto (Fig. 2A).

Mediante inmunohistoquímica se observó expresión de CRT de manera abundante y homogénea en el trofoblasto y en las células endoteliales fetales, mientras que en el citotrofoblasto se presentó un patrón heterogéneo (Fig. 2B).
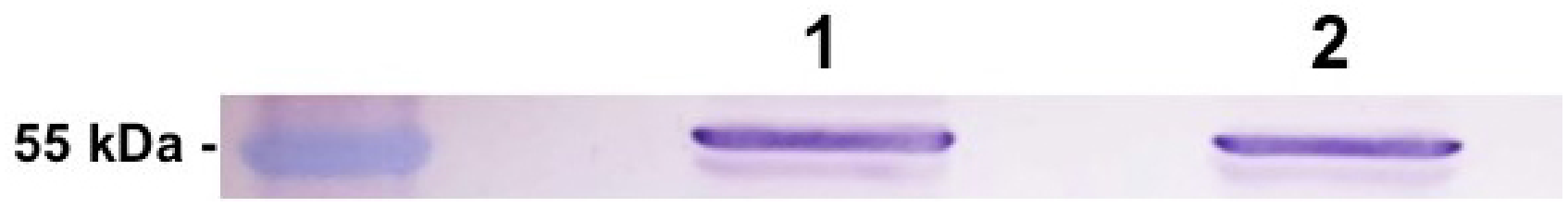

Fig. 1. Presencia de Calreticulina en placenta canina en un procedimiento de western blot. La membrana fue incubada con un suero inmune policlonal anti-CRT humana 1:1000. 1 y 2. Extracto de proteína total de placenta canina $(30 \mu \mathrm{g})$.
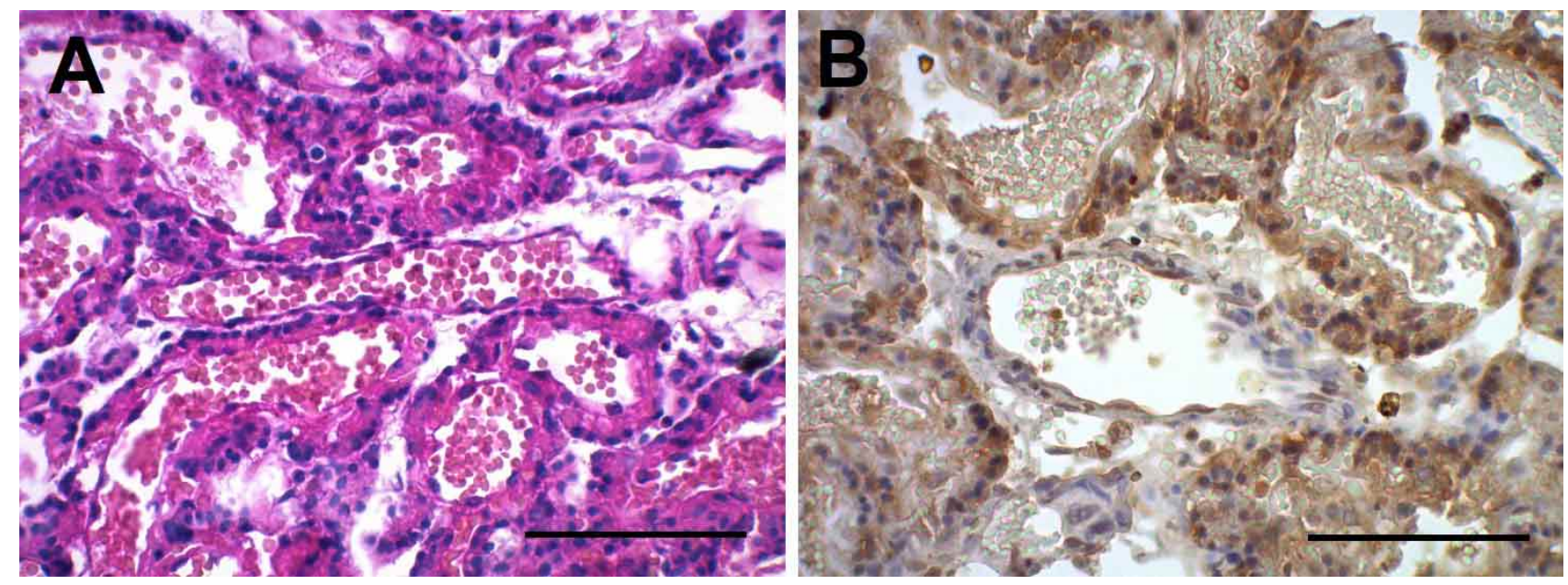

Fig. 2. Expresión de Calreticulina en placenta canina. A: Corte seriado de $5 \mu \mathrm{m}$ de placenta canina teñida con Hematoxilina-Eosina (400x). B: Inmunohistoquímica de placenta canina, la que fue incubada con suero inmune de conejo anti-CRT humana 1:1000 (400x). La barra equivale a $100 \mu \mathrm{m}$.

\section{DISCUSIÓN}

Se detectó la presencia de CRT canina con un peso molecular aparente de $62 \mathrm{kDa}$ (Fig. 1). Estos resultados concuerdan con los de Houen \& Koch, quienes mediante western blot y con un antisuero anti-CRT humana detectaron CRT en placenta humana con un peso molecular aparente de 60 $\mathrm{kDa}$, secuenciándola posteriormente. Si bien el peso molecular teórico de CRT es de $42 \mathrm{kDa}$, su punto isoeléctrico de 4.65 hace que la molécula migre a 60-62 kDa al ser analizada mediante SDS-PAGE (Pekáriková et al., 2010). Dado que CRT es una proteína altamente conservada y que existe alta homología entre CRTs de distintas especies mamíferas (Michalak et al.), era esperable que el suero policlonal de conejo anti CRT humana reconociera la molécula canina por reacción cruzada. 
CRT representa aproximadamente el 0,1\% de las proteínas placentarias caninas totales. Esto corresponde a una cantidad bastante alta si se la compara con otros tejidos en los que se ha detectado CRT, hecho que coincide con lo reportado para CRT, en placenta humana, por Højrup et al. y Gu et al.

En la placenta canina de término las vellosidades fetales revestidas por trofoblasto están en íntimo contacto con los vasos sanguíneos maternos (Fig. 2A), presentando diferencias parciales al compararlo con la placenta humana, donde la sangre materna baña a las vellosidades libres fetales revestidas por el trofoblasto.

CRT se expresa preferentemente en el trofoblasto de la placenta canina (Fig. 2B), situación que coincide con lo observado por Castillo et al. para la proteína humana, donde CRT se expresa principalmente en el sinciotrofoblasto y en la decidua basal. Esto da cuenta que, pese a que las placentas de origen canino y humano poseen diferencias morfológicas e histológicas, el patrón de expresión de CRT no varía, pudiendo inferirse que la presencia conservada de la proteína en distintas especies se condice con un importante rol en este tejido durante el embarazo/preñez.

Además de identificarse CRT en placenta humana (Houen \& Koch), se ha investigado sus niveles en sangre de mujeres embarazadas y se ha visto que éstos se encuentran elevados al compararlos con mujeres no embarazadas (Gu et al.), coincidiendo con lo observado por Cheng et al. en ratas. Este aumento de CRT sanguíneo indicaría que la proteína es necesaria para uno o varios procesos fisiológicos en el embarazo.

Por otra parte, se han investigado los niveles de CRT en patologías asociadas al embarazo como lo son la preeclampsia y la restricción de crecimiento fetal. En la preeclampsia, aumentan en los niveles de CRT en placenta al compararlo con embarazos normotensos, mientras que el caso de la restricción del crecimiento fetal no se han detectado niveles de CRT elevados en placentas humanas y de ratas (Crawford et al.). Aunque la pre-eclampsia es una patología exclusiva de humanos y primates (Crosley et al., 2013), CRT podría tener algún rol en otras patologías del embarazo. Más aun, futuros estudios podrían considerar los niveles de CRT en sangre o placenta como marcadores de patologías asociadas al embarazo.

Estudios más recientes han reportado que CRT se encuentra en mayor cantidad en el endometrio de ratonas preñadas versus no preñadas, y que los niveles más altos de la proteína se detectan el día 4 que corresponde al día de la implantación del blastocisto. Esto estaría dado por la regulación que ejerce CRT sobre las integrinas que guían el proceso de adhesión del blastocisto (Cheng et al.). Esto coincide con los resultados obtenidos por Yamamoto et al. (2017) en humanos, quienes describen que CRT se expresa en gran cantidad en en el trofoblasto y proponen que esta proteína regularía la invasión del trofoblasto en la decidua basal mediante mecanismos similares a los descritos por Cheng et al. Se requieren más estudios para determinar si efectivamente CRT regula los procesos de implantación del blastocisto, invasión del trofoblasto u otros aún desconocidos, que permiten llevar a cabo un embarazo exitoso.

Finalmente, los resultados de este trabajo indican que: i) La placenta canina es una buena fuente de CRT; ii) La elevada cantidad de CRT en placenta canina se condice con una función importante en este tejido; iii) CRT presenta un patrón de expresión en placenta canina similar al de humanos, lo que predice funciones importantes, al mantenerse conservada en distintas especies mamíferas.

ROSAS, C. C.; WEINBERGER, S. K.; MALDONADO, F. I. ; LEMUS, A. D. \& FERREIRA, V. A. Calreticulin expression in canine placenta. Int. J. Morphol., 37(4):12941298, 2019,

SUMMARY: The placenta is an embryonic organ present in mammals, whose main functions are the exchange of nutrients and gases and to protect the fetus from potential immune damage mediated by paternal and maternal allogeneic differences in the Major Histocompatibility Complex. Several proteins associated with its function have been described, being Calreticulin one of them. Although there are studies on the presence of Calreticulin in human placenta, there are no reports of this protein in canine placenta. Samples from canine placenta were obtained, proteins extracted and Calreticulin was subsequently detected by western blot and immunohistochemistry. The results showed the presence of Calreticulin in canine placenta with an apparent molecular weight of $60 \mathrm{kDa}$, in agreement with the results from other authors. The immunohistochemical analysis showed that canine Calreticulin is present mainly in the trophoblast of the villi, and there is no difference in its localization when compared with a blood-filled placenta such as human one, despite its morphological and histological differences. We also propose a standardized protocol for the extraction of Calreticulin from placenta, given its abundant expression in this organ. Future studies are aimed at elucidating possible roles of this protein in placenta.

\section{KEY WORDS: Calreticulin; Placenta;} Trophoblast. 


\section{REFERENCIAS BIBLIOGRÁFICAS}

Arosa, F. A.; de Jesus, O.; Porto, G.; Carmo, A. M. \& de Sousa, M. Calreticulin is expressed on the cell surface of activated human peripheral blood $\mathrm{T}$ lymphocytes in association with major histocompatibility complex class I molecules. J. Biol. Chem., 274(24):16917-22, 1999.

Bradford, M. M. A rapid and sensitive method for the quantitation of microgram quantities of protein utilizing the principle of protein-dye binding. Anal. Biochem., 72:248-54, 1976.

Castillo, C.; Ramirez, G.; Valck, C.; Aguilar, L.; Maldonado, I.; Rosas, C.; Galanti, N.; Kemmerling, U. \& Ferreira, A. The interaction of classical complement component $\mathrm{C} 1$ with parasite and host calreticulin mediates Trypanosoma cruzi infection of human placenta. Plos Negl. Trop. Dis., 7(8):e2376, 2013.

Cheng, S. Q.; He, J. L.; Dong, Y. L.; Liu, X. Q.; Ding, Y. B.; Gao, R. F.; Tan, Y.; Ye, Q.; Tian, Z. L. \& Wang, Y. X. Characterization of calreticulin expression in mouse endometrium during embryo implantation. Biol. Res., 42(4):505-16, 2009.

Crawford, K. E.; Kalionis, B.; Stevenson, J. L.; Brennecke, S. P. \& Gude, N. M. Calreticulin has opposing effects on the migration of human trophoblast and myometrial endothelial cells. Placenta, 33(5):416-23, 2012.

Crosley, E. J.; Elliot, M. G.; Christians, J. K. \& Crespi, B. J. Placental invasion, preeclampsia risk and adaptive molecular evolution at the origin of the great apes: evidence from genome-wide analyses. Placenta, 34(2):127-32, 2013.

Flieguel, L.; Burns, K.; MacLennan, D. H.; Reithmeier, R. A. F. \& Michalak, M. Molecular cloning of the high affinity calcium-binding protein (calreticulin) of skeletal muscle sarcoplasmic reticulum. J. Biol. Chem., 264(36):21522-8, 1989.

Gelebart, P.; Opas, M. \& Michalak, M. Calreticulin, a Ca2+-binding chaperone of the endoplasmic reticulum. Int. J. Biochem. Cell Biol., 37(2):260-6, 2005 .

Gold, L. I.; Eggleton, P.; Sweetwyne, M. T.; Van Duyn, L. B.; Greives, M. R.; Naylor, S. M.; Michalak, M. \& Murphy-Ulrich, J. E. Calreticulin: non-endoplasmic reticulum functions in physiology and disease. FASEB J., 24(3):665-83, 2010.

Gu, V. Y.; Wong, M. H.; Stevenson, J. L.; Crawford, K. E.; Brennecke, S. P. \& Gude, N. M. Calreticulin in human pregnancy and pre-eclampsia. Mol. Hum. Reprod., 14(5):309-15, 2008.

Gudea, N. M.; Roberts, C. T.; Kalionisa, B. \& King, R. G. Growth and function of the normal human placenta. Thromb. Res., 114(5-6):397407, 2004.

Højrup, P.; Roepstorff, P. \& Houen, G. Human placental calreticulin characterization of domain structure and post-translational modifications. Eur. J. Biochem., 268(9):2558-65, 2001.

Houen, G. \& Koch, C. Human placental calreticulin: purification, characterization and association with other proteins. Acta Chem. Scand., 48(11):905-11, 1994.

Kisters, K.; Barenbrock, M.; Louwen, F.; Hausberg, M.; Rahn, K. H. \& Kosch, M. Membrane, intracellular, and plasma magnesium and calcium concentrations in preeclampsia. Am. J. Hypertens., 13(7):765-8, 2000.

Mesaeli, N.; Nakamura, K.; Zvaritch, E.; Dickie, P.; Dziak, E.; Krause, K. H.; Opas, M.; MacLennan, D. H. \& Michalak, M. Calreticulin is essential for cardiac development. J. Cell Biol., 144(5):857-68, 1999.

Michalak, M.; Corbett, E. F.; Mesaeli, N.; Nakamura, K. \& Opas, M. Calreticulin: one protein, one gene, many functions. Biochem. J., 344 Pt. 2:281-92, 1999.

Milner, R. E.; Baksh, S.; Shemanko, C.; Carpenter, M. R.; Smillie, L.; Vance, J. E.; Opas, M. \& Michalak, M. Calreticulin, and not calsequestrin, is the major calcium binding protein of smooth muscle sarcoplasmic reticulum and liver endoplasmic reticulum. J. Biol. Chem., 266(11):7155-65, 1991.
Moffett, A. \& Loke, C. Immunology of placentation in eutherian mammals. Nat. Rev. Immunol., 6(8):584-94, 2006.

Moore, K. L.; Persaud, T. V.; \& Torchia, M. G. Embriología clínica. 10ª ed. Barcelona, Elsevier, 2016.

Pekáriková, A.; Sánchez, D.; Palová-Jelínková, L.; Simsová, M.; Benes, Z.; Hoffmanová, I.; Drastich, P.; Janatková, I.; Mothes, T.; TlaskalováHogenová, H.; et al. Calreticulin is a B cell molecular target in some gastrointestinal malignancies. Clin. Exp. Immunol., 160(2):215-22, 2010.

Roa, I.; Smok, C., \& Prieto, R. Placenta: compared anatomy and histology. Int. J. Morphol., 30(4):1490-6, 2012.

Yamamoto, M.; Ikezaki, M.; Toujima, S.; Iwahashi, N.; Mizoguchi, M.; Nanjo, S.; Minami, S.; Ihara, Y. \& Irio, K. Calreticulin Is involved in invasion of human extravillous trophoblasts through functional regulation of integrin b1. Endocrinology, 158(11):3874-89, 2017.

\author{
Dirección para correspondencia: \\ Arturo Ferreira, DVM, PhD. \\ Professor of Immunolgy \\ Programa Disciplinario de Inmunología \\ Facultad de Medicina, ICBM \\ Universidad de Chile \\ Santiago \\ CHILE
}

Email: aferreir@med.uchile.cl

Recibido : 28-03-2019

Aceptado: 21-06-2019 